\title{
LOS FACTORES DETERMINANTES DEL VOTO: POR QUÉ EL VOTO OBLIGATORIO NO ES LA SOLUCIÓN AL ABSTENCIONISMO *
}

\section{The determinant factors of voting: why compulsory voting is not the solution to electoral abstentionism}

José Fernando Flórez Ruiz**, Ph. D.

Recepción: 12 de abril de 2018. Aceptación: 27 de noviembre de 2018.

DOI: http://dx.doi.org/10.21017/Rev.Repub.2019.v27.a73

"A man in no less slave because he is allowed to choose a new master once in a term of years».

Lysander Spooner (1870, «No Treason»)

\section{RESUMEN}

Este artículo hace una revisión sistemática de la literatura sobre los factores determinantes del voto. A partir de ella, plantea una nueva clasificación que los categoriza como factores individuales y contextuales, que pueden a su vez consistir en variables demográficas, psicológicas, macrodemográficas, políticas e institucionales. El análisis de la dinámica causal del voto pone en evidencia que, lejos de ser un «acto natural», la acción de votar presenta altos costos para el potencial elector. Desde esa perspectiva, defiendo la hipótesis normativa de que el abstencionista no debería ser satanizado como un «mal ciudadano»,

* Este artículo es un producto del proyecto de investigación Abstencionismo del «mal ciudadano»: una revisión sobre los determinantes del voto, del Grupo Derecho Laboral, Económico y Estado de la Corporación Universitaria Republicana.

** Abogado de la Universidad Externado de Colombia, especialista en Derecho Constitucional, magíster en Políticas Públicas y doctor (Ph. D.) en Ciencia Política por la Universidad París II Panthéon-Assas. Es el autor de los libros Todo lo que la democracia no es y lo poco que sí: defensa de una concepción democrática realista (Universidad Externado de Colombia, 2015, 2. ${ }^{a}$ reimpresión en 2017) y Voter sans élire: le caractère antidémocratique de la réélection présidentielle immédiate en Amérique Latine, 19942018 (Institut Universitaire Varenne Collection des Thèses, 2018). Coautor del libro El pueblo contra sí mismo: el plebiscito por la paz en Colombia y los límites de la democracia (Ediciones Nueva Jurídica, 2018) y editor del libro Derechos humanos y no humanos de última generación: la superación del antropocentrismo en el derecho constitucional (Tirant Lo Blanch, 2019). Actualmente es Director de Planeación en la Registraduría Nacional del Estado Civil. Correo electrónico: florezjose@gmail.com, jfflorez@registraduria.gov.co, @florezjose en Twitter. 
ya que el abstencionismo es la consecuencia natural de las dificultades para votar, así como de las asimetrías en la información que tienen las personas y su interés desigual en la política, que es un gesto de libertad. Por último, advierto sobre el peligro de forzar a los ciudadanos a votar para aumentar artificialmente la tasa de participación, pues existe evidencia empírica de que el voto obligatorio conduce a una decisión electoral menos informada que deteriora la calidad democrática.

Palabras clave: abstencionismo, comportamiento electoral, democracia, elecciones, voto obligatorio.

\begin{abstract}
A systematic review of the literature on the determinants of voting leads to classifying them as individual and contextual factors, which, in turn, can consist of demographic, psychological, macro-demographic, political and institutional variables. This analysis shows that, far from being a "natural act", the action of voting has high costs for the potential voter. From this perspective, the abstentionist should not be demonized as a "bad citizen". Furthermore, forcing citizens to vote in order to increase voter turnout could be counterproductive, since there is empirical evidence that compulsory voting produces less informed electoral decisions that deteriorates the quality of democracy.
\end{abstract}

Key words: compulsory voting, elections, electoral behavior, democracy, voter turnout.

\title{
1. INTRODUCCIÓN
}

Este artículo de revisión bibliográfica tiene un doble objetivo. En primer lugar, cumple una función didáctica porque es una síntesis del estado actual del conocimiento sobre el comportamiento electoral y los principales factores que mueven a las personas a votar. Para ese propósito, sistematiza los hallazgos de la literatura especializada sobre las variables determinantes del voto, mediante una nueva clasificación que se construyó a partir de una búsqueda selectiva que privilegió el análisis de los trabajos con enfoque empírico -tanto los clásicos como los más recientes- en la bibliografía nacional y comparada.

El segundo objetivo del artículo es desmitificador y, por ende, crítico. A partir de la sistematización del conocimiento sobre los móviles del voto que hace en la tercera parte, pone en entredicho dos lugares comunes que han venido haciendo carrera en algunos sectores de la teoría democrática menos realista. El primero de ellos es la creencia de que el voto es un acto casi "natural" en el humano y por lo tanto las altas tasas de abstencionismo que registra el mundo democrático son un fenómeno anómalo. A ese lugar común se apareja el juicio 
de valor negativo de que los abstencionistas son «malos ciudadanos», es decir, una suerte de ovejas negras de la sociedad a las que cabe censurar e incluso sancionar por no interesarse en la política electoral. Para refutar esa primera creencia, se visibilizan en la segunda parte los enormes costos que tiene el gesto de votar, que por lo general pasan inadvertidos debido a la normalización del mito democrático.

El segundo lugar común que problematizo es la creencia de que el voto obligatorio sería la «solución» al «problema» del abstencionismo. Las comillas se justifican porque considerar que el abstencionismo es un problema, sin más, es una petición de principio. En la última parte explico las razones por las cuales forzar a la gente a votar mediante leyes de voto obligatorio, aunque eleva significativamente la tasa de participación electoral, resulta contraproducente porque empeora la calidad de la participación democrática.

En correspondencia con todo lo anterior, la principal pregunta de investigación que se hace este trabajo es si el abstencionismo electoral es realmente un «problema» para la democracia, o si es más bien la consecuencia normal de la libertad de las personas y de los obstáculos económicos que enfrentan para votar. La hipótesis de la que parto y parece verificarse según se desprende de la revisión bibliográfica efectuada, es que el abstencionismo no es necesariamente un problema, sino un filtro natural en las democracias liberales que cumple la función de propiciar que quienes se interesan más por la política electoral y están mejor informados sobre ella sean quienes votan con mayor frecuencia como resultado de decisiones electorales más racionales y con ello significativas. La idea que subyace a esa conclusión es que una mayor participación electoral no es necesariamente sinónimo de robustez democrática.

\section{EL COSTO DE VOTAR. POR QUÉ EL VOTO NO ES UN ACTO NATURAL}

El domingo 2 de octubre de 2016 los colombianos recibieron con enorme sorpresa la noticia de que el «No» había triunfado en el plebiscito por la paz. Mediante ese mecanismo, el gobierno del presidente de Juan Manuel Santos sometió a refrendación popular el «Acuerdo Final para la Terminación del Conflicto y la Construcción de una Paz Estable y Duradera», que había firmado seis días atrás con el grupo guerrillero FARC para ponerle fin a un conflicto armado interno de más de medio siglo de duración, que dejó un saldo de 220.000 muertos (Flórez et al., 2018).

La sorpresa con el resultado electoral se produjo por partida doble: no solo porque en el plebiscito ganó el «No» con el 50,21 \% de los votos (frente al 
49,78 \% del «Sí»), en contra de lo que habían pronosticado todas las encuestas, sino porque, según cifras de la Registraduría Nacional, la participación alcanzó apenas 13.066.047 votantes, lo que significó una abstención electoral del 62,6 \%, la más alta registrada en una elección de alcance nacional desde la Constitución de 1991.

¿Cómo es posible que, en un evento de tanta trascendencia histórica como la posibilidad de terminar una guerra interna, apenas el 37,4 \% de los colombianos habilitados para votar lo hayan hecho? ¿Por qué más de 21 millones de individuos (el potencial electoral para esa elección era de 34.899 .945 votos) se abstuvieron de participar en el que tal vez será el evento democrático más importante del siglo XXI en Colombia? En definitiva, ¿por qué se abstienen de votar las personas?

Lo primero que compete refutar a este respecto es la falsa idea de que salir a votar el día de elecciones es casi una inclinación «natural» en los humanos. Jane Jacobs $(1969$, p. 121) señaló con agudeza que «buscar las causas de la pobreza es un callejón sin salida intelectual porque la pobreza no tiene causas. Solo la prosperidad tiene causas» ${ }^{1}$.

Algo similar ocurre con el voto. De la misma manera en que no hay que explicar por qué la gente es pobre, pues la pobreza (la ausencia de riqueza) es un estado natural que solo se ve alterado gracias al desarrollo económico que genera riqueza (como sucede con el frío, que no es el resultado de ningún proceso activo, sino apenas es la ausencia de calor, para continuar con la brillante analogía de Jacobs), no hay que preguntarse por qué en los regímenes democráticos la gente no vota, pues el abstencionismo no tiene causas, sino más bien por qué habría de hacerlo cuando votar es un acto costoso en términos de tiempo, energía y dinero.

Ciertamente, el acto de votar implica costos y la superación de barreras para el potencial elector. Esos costos y barreras incluyen: hacer el registro electoral; localizar y transportarse hasta los puestos de votación; esperar en la fila de la mesa de votación; sacar el tiempo para ir a votar en medio de las obligaciones personales y profesionales; a veces, incluso, luchar contra el mal clima el día de la votación; pero, sobre todo, votar implica informarse sobre los candidatos, sus propuestas, los principales asuntos de interés en cada elección y las complejas

1 'To seek 'causes' of poverty...is an intellectual dead end because poverty has no causes. Only prosperity has causes. Analogically, heat is a result of active processes; it has causes. But cold is not the result of any process; it is only the absence of heat. Just so, the great cold of poverty and economic stagnation is merely the absence of economic development. It can be overcome only if the relevant economic processes are in motion". 
reglas institucionales que la rodean, lo cual requiere gastar una cantidad significativa de tiempo que la mayoría de las personas no está dispuesta a invertir en política.

Una vez que se explicita el costo que tiene para cualquier ciudadano el acto de votar, es más fácil comprender por qué, en las democracias que carecen de voto obligatorio (esto es, aquellas que le ponen un costo institucionalizado más o menos elevado al incumplimiento de la obligación de votar), el abstencionismo de alrededor de la mitad del electorado es la regla y no la excepción.

Díaz et al. (2017, p. 51) estimaron el promedio de participación electoral en las democracias con voto voluntario en $52,4 \%$, frente a $75,8 \%$ en países con voto obligatorio. Blais (2011) calculó la participación electoral mundial promedio en un $70 \%$. Según el International Institute for Democracy and Electoral Assistance (2013), la tasa global de participación electoral ha caído en más de un $10 \%$ entre 1960 y 2000. En una observación que cubrió 27 países entre 1978 y 2003, Kostadinova y Power (2007) evidenciaron que, en las democracias nacidas de la tercera oleada de democratización, tanto en Latinoamérica como en Europa del Este, los niveles de participación iniciales cayeron al menos hasta la cuarta elección luego de la elección fundadora. Los autores atribuyen ese efecto a la «hipótesis de la elección fundadora», según la cual la participación electoral debe declinar gradualmente después de la elección que inaugura una democracia, debido a la paulatina pérdida de entusiasmo del electorado con el nuevo régimen a medida que los ciudadanos se dan cuenta de que las elecciones no son una panacea.

El abstencionismo electoral generalizado se explica en gran medida porque el voto es un fenómeno que responde esencialmente a una racionalidad económica. Desde esa perspectiva, hace cuatro décadas Edward Tufte (1978, p. 65) escribió: «cuando uno piensa en economía, piensa en elecciones; cuando uno piensa en elecciones, piensa en economía». No es una coincidencia que, más de sesenta años después de haber sido publicado, el libro clásico de Anthony Downs (1957), An Economic Theory of Democracy, sea el más citado en el Handbook of Political Science (Lehingue, 2011, p. 185).

De la teoría o enfoque económico del voto se desprenden esencialmente dos conclusiones. La primera es que el elector decide si vota o no y en qué sentido lo hace con base en incentivos económicos, es decir, respondiendo a una racionalidad económica en la que evalúa los costos y beneficios del acto de votar. A esa aproximación se le conoce en el campo del comportamiento electoral como «teoría de la elección racional» (Abitbol y Botero, 2005). La segunda conclusión es que, cuando votan, los electores deciden premiar con la reelección a los gobernantes cuya gestión puede reivindicar un buen desempeño económico, y 
castigarlos eligiendo a otros candidatos cuando perciben que la gestión económica ha sido negativa (Navia y Osorio, 2015).

En el siguiente acápite, propongo una sistematización de las variables que aumentan o disminuyen la probabilidad de que las personas voten, según la evidencia empírica más reciente disponible. Esas variables pueden clasificarse como factores personales (2.1), que a su vez pueden ser demográficos (2.1.1) y psicológicos (2.1.2); y factores contextuales (2.2) que, a su vez, pueden categorizarse como macrodemográficos (2.2.1), políticos (2.2.2) e institucionales (2.2.3).

\section{UNA SISTEMATIZACIÓN DE LOS FACTORES DETERMINANTES DEL VOTO}

El modelo de factores determinantes o predictores del voto que presento a continuación no aspira a la exhaustividad. El voto es un fenómeno complejo y multicausal en el que pueden influir parámetros adicionales a los aquí estudiados. Piénsese, por ejemplo, en el enorme impacto que tiene en el resultado de las elecciones presidenciales el hecho de que el presidente en ejercicio aspire a la reelección inmediata.

Cuando el presidente en el poder busca la reelección es imposible derrotarlo, salvo en escenarios de colapso económico, pues el uso de los recursos del Estado y el abuso del poder presidencial con fines electorales le otorga una ventaja exorbitante que lo convierte en un competidor invencible para los demás candidatos (Flórez, 2018b).

En efecto, la presencia en el proceso electoral latinoamericano de un candidato que también ejerce el cargo de presidente aumenta la diferencia promedio entre el primer y el segundo puesto en la contienda electoral en un 15,4\% de los votos. Sin embargo, el crecimiento de un punto en el índice de calidad democrática Polity IV hace que la diferencia de votos entre el ganador y el segundo candidato se reduzca en un $2,87 \%$. Esto indica que el hecho de ser candidato mientras se ocupa la Presidencia aumenta la probabilidad de ganar las elecciones, pero, cuanto mayor es la calidad de la democracia, disminuye la diferencia entre el ganador y el segundo porque se reequilibra la competencia (Flórez, 2018a).

Otras variables absolutamente contingentes, que en principio no deberían importar porque no dependen en lo absoluto del desempeño del Gobierno, como el resultado de un partido de fútbol, baloncesto, o cualquier otro deporte de gran importancia nacional, pueden llegar a tener un impacto significativo en la decisión electoral. 
Healy et al. $(2009,2010)$ demostraron que, en Estados Unidos, entre 1964 y 2008 , la euforia y felicidad producidas por el triunfo del equipo local de fútbol americano dentro de las dos semanas previas a las elecciones tuvo un efecto en el comportamiento electoral favorable al statu quo: incrementó la votación del presidente, gobernador o senador que aspiraba a la reelección en un 1,61 \%, e incluso más cuando se trató de equipos que tienen las mayores fanaticadas (en estos casos el aumento llegó a ser de entre un 2,3 y un 2,42 \%). Esos resultados fueron corroborados por la observación de la influencia de los partidos de baloncesto en la popularidad del presidente Barack Obama en 2009, cuya favorabilidad aumentó en 2,3 \% en los lugares donde el equipo local había ganado.

Desde esa perspectiva, y teniendo en cuenta que, según una encuesta realizada por The New York Times (2014), Colombia es el país de Latinoamérica que le da mayor importancia al fútbol, tiene sentido pensar que el triunfo de la Selección Colombia contra Grecia (3-0) en la primera fase del Mundial de Fútbol Brasil 2014, un día antes de la segunda vuelta presidencial (que tuvo lugar el 15 de junio de 2014), pudo tener un impacto favorable de alrededor de 300.000 votos en el triunfo del presidente-candidato Juan Manuel Santos (La Silla Vacía, 2014).

Sin embargo, ese efecto eufórico es pasajero. Cuando Brasil sufrió contra Alemania la peor derrota (7-1) de su historia en los mundiales, el 8 de julio de 2014, en el denominado «Mineiratzen», se especuló que la candidatura a la reelección de la presidenta Dilma Roussef estaba sepultada. Su triunfo en la segunda vuelta, el 26 de octubre, probó que luego de algunos meses el electorado ya había «perdonado» la humillación futbolera.

\subsection{Factores individuales}

Las características personales juegan un papel determinante en la proclividad de las personas a votar. La juventud, el nivel bajo de ingresos y la poca escolaridad, por ejemplo, son variables que contribuyen directamente al abstencionismo en la mayoría de las democracias. Por su parte, la condición de funcionario público, así como la militancia en partidos políticos y la pertenencia a diferentes asociaciones humanas, suelen impactar positivamente la participación electoral debido al interés elevado en política al que están asociadas.

\subsubsection{Variables demográficas}

La edad, el ingreso y el nivel educativo son las tres principales variables personales que impactan positivamente la proclividad a votar. El parámetro de mayor peso en la participación electoral es la educación (Wolfinger y Rosenstone, 1980), 
que a su vez suele estar correlacionada con un mayor ingreso: hay evidencia transnacional robusta de que el abstencionismo es más alto en los países menos ricos (Blais, 2006).

La explicación de que las personas más educadas y de mayor ingreso voten con más frecuencia radica en el hecho de que la información y el interés en política son acciones sociales que implican cierta sofisticación intelectual y tiempo libre. Dicho de otra manera, la política es un «lujo» que solo se pueden permitir las personas con un mínimo nivel cultural, que tienen sus necesidades básicas satisfechas (Leighley y Nagler, 2013; Lijphart, 1997). Con una mayor educación, también mejoran las capacidades cognitivas y se reducen los costos de estar informado sobre política y elecciones (Maldonado, 2011).

El envejecimiento está asociado a una mayor proclividad a ejercer el sufragio por cuanto la formación del votante funcional toma algún tiempo. La baja participación de los «primivotantes» (personas entre 18 y 25 años) obedece al tiempo y experiencia que normalmente requiere la formación del ciudadano electoralmente activo. El hábito de votar es típico del adulto y la transición de la adolescencia a la madurez política es un proceso paulatino (Goerres, 2007).

Otras características como la condición de funcionario público, estar casado y asistir con regularidad a servicios religiosos también aumentan la proclividad a votar. La mayor participación entre servidores públicos se explica por la politización incrementada que experimentan las personas que trabajan con el Estado, fuera del impacto positivo que normalmente tiene el empleo formal en la participación electoral (Maldonado, 2011), al aumentar el ingreso y facilitar la integración social.

No es que el matrimonio y los dioses sean «demócratas» $\mathrm{y}$, por tanto, particularmente funcionales al voto. El impacto positivo de la vida de pareja y la religiosidad sobre la participación electoral es indirecto y se explica por una característica que comparten las personas mejor educadas, mayores, más religiosas, más ricas y casadas: todas «tienden a estar integradas en la sociedad» (Blais, 2000: 52). Esta característica común permite inferir que el gesto de votar expresa un sentido de pertenencia del individuo hacia la comunidad, por lo que sería un hábito estrechamente vinculado a la sociabilidad de las personas.

Por último, el género femenino puede ser un predictor de la abstención en democracias con un machismo muy preponderante. Power y Roberts (1995) encontraron que, en Brasil, el porcentaje de participación femenina en la fuerza laboral está positivamente relacionado con una mayor tasa de votación. En otra investigación, Lehoucq y Wall (2004) observaron que el aumento del 
número de mujeres inscritas para votar también impactó positivamente la tasa de participación en 330 municipios de Guatemala. De estos datos se puede deducir que la participación electoral responde a la importancia de la mujer en la sociedad. Sin embargo, una vez que la mujer alcanza un umbral importante de integración social gracias a la liberalización, el género pierde significancia estadística como predictor del voto.

En definitiva, las características del votante obran como factores predictores del comportamiento electoral (Perea, 2002), pero las tendencias y el peso relativo de las variables pueden cambiar en función del contexto (Blais, 2006). Topf (1995), por ejemplo, testeó tres variables en los países de Europa Occidental y encontró que allí la educación y el género no tenían mayor relevancia, mientras que la edad sí mostró un efecto significativo. De ahí la necesidad de dibujar el perfil socioeconómico específico del votante de cada país o circunscripción electoral objeto de análisis.

\subsubsection{Variables psicológicas}

Existe evidencia de que el votante funcional empieza a formarse desde la infancia. La participación política es una forma de expresión e interacción social adulta que se ve favorecida por ciertas formas de socialización y habilidades que se desarrollan durante la infancia. En otras palabras, en el niño social de hoy está el germen del ciudadano del mañana. Dos estudios suministran evidencia empírica sobre esta premisa.

Rojas y Hopke (2010) probaron que, en Colombia, la «comunicación autoritaria» durante la niñez, padecida por individuos que tuvieron dificultades en la infancia para expresar sus opiniones y disentir de figuras de autoridad en su familia o colegio, hizo que para estas personas fuera más difícil expresarse políticamente como adultos. En palabras de los autores, «la comunicación autoritaria en la infancia está relacionada positivamente con la autocensura de las opiniones políticas como adulto», entre las cuales cabe incluir el voto.

La muestra utilizada por este estudio comprendió 1.009 personas, representativas de la población colombiana urbana adulta. Y la variable dependiente de «autocensura» fue medida mediante seis ítems, que le pedían al encuestado manifestar su grado de acuerdo con algunas afirmaciones sobre la dificultad para expresar sus opiniones frente a personas que probablemente no comparten su forma de pensar, utilizando una escala de 0 a 5, en la que 0 expresaba «total desacuerdo» y 5, «total acuerdo». Por su parte, la variable explicativa de «comunicación autoritaria en la infancia» se estableció mediante dos ítems que indagaban por la comunicación en el campo familiar y escolar durante la infancia, preguntándoles a las personas qué tan difícil había sido para ellas expresar 
desacuerdo frente a sus padres, familiares y profesores, en una escala en la que 0 era «muy fácil» y 5 , «muy difícil».

Más recientemente, Holbein (2017) logró evaluar empíricamente el impacto del desarrollo de habilidades psicosociales durante la niñez en la participación electoral en la edad adulta. Valiéndose de la base de datos del proyecto estadounidense «Fast Track»², que intervino desde 1992 una muestra de 445 niños que estaban apenas terminando el kindergarten para aumentar su capacidad de regulación emocional y sus capacidades sociocognitivas, el autor emparejó la identidad de los niños tratados con su registro electoral una vez alcanzaron la edad de entre 26 y 29 años, en julio de 2014.

Esta investigación encontró que los niños que se sometieron al programa completo de capacitación psicosocial (que tenía seis componentes: un currículo formal guiado por un instructor, visitas al hogar, tutorías, grupos de entrenamiento a los padres, grupos de amistad y trabajo en parejas) registraron en la edad adulta una participación electoral en promedio un 11,1 \% superior a la del grupo de control.

Las habilidades psicosociales también son conocidas como «habilidades no cognitivas». Mientras que las habilidades cognitivas involucran la aplicación de lógica, razonamiento y memoria, las habilidades psicosociales se suelen clasificar en dos categorías: las que implican autorregulación (como, por ejemplo, la autosuficiencia, la capacidad de posponer la gratificación, la perseverancia, la regulación de las emociones y el control comportamental) y las que envuelven habilidades sociales (como la habilidad de trabajar con otros productivamente, comunicarse, construir amistades y resolver problemas de grupo).

Tradicionalmente, la formación política escolar se ha enfocado en el desarrollo de habilidades en el nivel cognitivo, tales como el estudio del diseño institucional y el conocimiento de la cultura e historia política de los países. El trabajo de Holbein señala que se necesita una reorientación de la socialización política de los niños para hacerla más integral y fortalecer así también sus habilidades psicosociales. El desarrollo de ambos tipos de competencias resulta relevante para facilitar la formación de ciudadanos más involucrados y activos desde la niñez, lo cual conduciría a una mayor participación electoral.

\subsection{Factores contextuales}

La literatura especializada ha identificado diversos factores del contexto en el cual se realizan las elecciones que predicen el comportamiento electoral. Como

2 http://fasttrackproject.org 
los estudios al respecto son numerosos y a veces ofrecen resultados ambiguos en función del contexto analizado, cabe recurrir a una revisión de la literatura especializada para identificar algunos consensos.

Geys (2006) hizo una revisión de la evidencia empírica presentada en 83 estudios y clasificó los macropredictores según sean variables de orden demográfico $^{3}$, político o institucional. Lo más interesante del metaanálisis (un «análisis de los análisis») efectuado por el autor belga es que le permitió establecer respecto de qué parámetros los resultados acumulados para la época del estudio (2006) eran estadísticamente concluyentes y apuntaban en la misma dirección. Veamos a continuación los principales resultados.

\subsubsection{Variables macrodemográficas}

La composición demográfica de un país o cualquier otra circunscripción electoral tiene una influencia decisiva en la proclividad a votar de los ciudadanos. Entre las cinco variables demográficas consideradas por Geys (2006), se cuentan el tamaño de la población, su concentración o densidad poblacional, su homogeneidad, la estabilidad demográfica y la participación previa. La última de estas variables puede considerarse demográfica porque hace referencia a la «cultura del voto».

La participación electoral efectivamente aumenta cuando la población es más pequeña, más estable, y las tasas previas de votación son más altas. Sin embargo, no es verificable una relación estadística significativa entre participación electoral, por un lado, y concentración y homogeneidad de la población, por el otro.

La mayor participación cuando la población es pequeña se produce como efecto de la «cercanía» (closeness) de las elecciones al elector: es más probable que los ciudadanos se interesen en la contienda electoral cuando perciben que la elección los concierne o «toca» más directamente. Esto tiene que ver con la idea de que el voto tendrá un mayor impacto en la elección cuando el número de votantes es menor. Piénsese, por ejemplo, en las elecciones para elegir los miembros del Consejo de Administración en los regímenes de propiedad horizontal. En esas elecciones participan microelectorados (los propietarios de los inmuebles) que se cuentan por decenas, o a lo sumo por centenas, conformados por personas directamente concernidas por los asuntos que definirán los ganadores de las elecciones (cambios al régimen de propiedad horizontal, fijación de la cuota de administración y mantenimiento de las zonas de uso común, de cuotas extraordinarias, entre otros). Por esa misma razón, la

3 Geys (2006) equivocadamente denomina a estas variables «socioeconómicas». Aquí corregimos el nombre por evidentes razones. 
participación tiende a ser más alta para elegir alcaldes que para elegir presidente, y todavía más para elegir alcaldes de municipios pequeños.

La estabilidad de la población es un parámetro que representa la cantidad de personas que viven de forma permanente en un lugar y se puede medir por la variable proxy del número de arrendatarios que allí existen. Cuando la población flotante de una circunscripción electoral es mayor, también lo es el desarraigo y, por ende, disminuye la participación electoral. Una medida racional para disminuir el abstencionismo sería, entonces, promover la adquisición de vivienda propia en la respectiva circunscripción. Un electorado de propietarios paga también más impuestos y, por consiguiente, tiende a interesarse más en política.

Persson y Rothstein (2015) encontraron una relación positiva entre el cobro de más impuestos directos y una mayor rendición de cuentas de los gobernantes, gracias al uso más frecuente de los mecanismos de control ciudadano. Para medirla, utilizaron el uso del marco legal disponible para controlar al personal político como variable proxy que indica un mayor control político ciudadano.

Por su parte, Percival et al. (2007) evaluaron empíricamente la relación entre carga tributaria, gasto público y participación en las elecciones locales de Estados Unidos. La conclusión general de los tres modelos de regresión presentados fue que existe evidencia robusta que soporta la principal hipótesis avanzada en el estudio: que «cuando los gobiernos estatales gastan más o cobran más impuestos, las apuestas electorales aumentan, lo que resulta en mayores niveles de participación electoral en las elecciones estatales» (p. 140).

La participación previa en las elecciones es otro predictor fiable de la futura participación. Coppock y Green (2015) señalan que «entre las generalizaciones empíricas más sólidas de la ciencia política está la observación de que las diferencias individuales en las tasas de participación electoral tienden a persistir en el tiempo». Esto se explica fundamentalmente porque el propio hecho de votar forma un hábito que se consolida paulatinamente. La «cultura del voto» no es otra cosa que un hábito colectivo que se ha formado y estabilizado en algunas sociedades democráticas.

La concentración y homogeneidad de la población, en contraste, no mostraron significancia estadística al agregar los estudios analizados. Con respecto a la concentración demográfica, se espera que reduzca la participación electoral con base en la premisa de que la urbanización conduce a un debilitamiento de los lazos interpersonales, las estructuras sociales primarias y el consenso sobre las normas (Hoffman-Martinot, 1994; Wirth, 1938). Por esa razón, las ciudades serían más individualistas y ello reduciría la «presión social» para votar 
en los lugares más densamente poblados, argumento que descansa sobre la idea de que votar es un deber cívico cuyo incumplimiento provoca una pérdida de prestigio social y tiene un costo en la reputación para el individuo abstencionista.

Por último, la hipótesis no verificada de que la homogeneidad de la población aumenta la participación electoral reposa sobre la creencia de que «la homogeneidad social es un prerrequisito de la cohesión de la comunidad» (Cohen, 1982: 259). En consecuencia, como la cohesión social aumenta la solidaridad de grupo (y de paso la «presión social»), la participación política en comunidades con un alto grado de homogeneidad socioeconómica, racial o étnica debería ser mayor.

\subsubsection{Variables políticas}

Dentro de los factores políticos fueron analizados la cercanía (o su contrario, la marginalidad) de las elecciones, el efecto del gasto en las campañas políticas y el nivel de fragmentación política en términos del número de partidos que participan en una elección. Geys (2006) encontró que las dos primeras variables están positivamente correlacionadas con la participación electoral, pero el efecto de la fragmentación política es ambiguo.

La cercanía (closeness) de las elecciones se refiere a su competitividad, medida por la brecha en puntos porcentuales entre la votación obtenida por el primero y el segundo candidato. Cuanto más «estrecha» sea la diferencia entre los dos primeros candidatos, más «cercana» o apretada estará la elección y el ciudadano tendrá la idea de que su voto tiene mayor probabilidad de afectar el resultado, de ser «decisivo» $y$, por ende, mayor será la participación. «Cuanto más apretada la elección, mayor la participación» (Blais, 2000: 30).

El impacto positivo en la participación electoral del mayor gasto en campañas se debe a que la inversión de dinero en política aumenta la visibilidad de los candidatos y de las elecciones en general, en particular por el gasto en publicidad y el mayor cubrimiento mediático que produce, al tiempo que reduce el costo de adquisición de información por parte de los electores (Dawson y Zinser, 1976; Chapman y Palda, 1983), todo lo cual aumenta la probabilidad de que voten. Cox y Munger (1989) señalan, además, que las campañas en contra del abstencionismo electoral ('get-out-the-vote' campaigns), aunque no necesariamente proveen información sobre los candidatos, sí estimulan el sentimiento de deber cívico en el votante y la participación.

Sin embargo, Ansolabehere et al. $(1994,1999)$ plantean la posibilidad de que las campañas negras o negativas tengan el efecto contrario, al reducir el apoyo al 
candidato atacado entre sus seguidores y alimentar una imagen negativa sobre la política en general, que desmoviliza a los potenciales electores.

La fragmentación partidista presenta efectos ambiguos en la literatura. No existe consenso sobre si un mayor número de partidos impacta positiva o negativamente la participación electoral. Es de esperarse un efecto positivo debido a que se enriquece la oferta política presentada al elector, lo que aumentaría la probabilidad de que se identifique con algún partido y, en consecuencia, de que vote (Seidle y Miller, 1976; Blais y Carty, 1990; Hansen, 1994). No obstante, Blais y Dobrzynska (1998) advierten que una mayor cantidad de partidos también incrementa la complejidad del sistema político, lo que hace más difícil para el elector tomar una decisión. Este aumento en los costos de información para el votante reduciría, entonces, la probabilidad de que asista a las urnas (Hoffman-Martinot, 1994).

\subsubsection{Variables institucionales}

En tercer lugar, las variables institucionales evaluadas por Geys (2006) se refieren al sistema electoral, las leyes de voto obligatorio, la presencia de elecciones concurrentes y los requisitos para la inscripción. Todos estos incentivos institucionales han mostrado un fuerte valor predictivo, pues el uso de métodos proporcionales de representación, el voto obligatorio, las elecciones realizadas el mismo día y los procedimientos de registro fáciles estimulan significativamente la participación. La explicación del impacto positivo de las dos últimas variables es que ambas reducen los costos de votar, lo que hace más fácil para el elector expresarse en las urnas.

La principal razón por la que un sistema electoral proporcional induce una mayor participación es que la desproporción entre los votos y las curules obtenidas que se presenta en un sistema electoral mayoritario (Ladner y Milner, 1999) lleva a los potenciales votantes a pensar que su voto no importa, tanto más si se trata de seguidores de partidos pequeños, con lo cual se incentiva el abstencionismo (Jackman, 1987). En un sistema electoral proporcional también es menos probable que existan circunscripciones electorales «no competitivas», es decir, en las que el ganador se pueda conocer de antemano. Esta situación les da a los partidos más incentivos para hacer campaña en todas las circunscripciones, lo que estimula la participación electoral (Blais y Carty, 1990).

El voto obligatorio, por su parte, aumenta la probabilidad de que la gente vote porque eleva el costo del abstencionismo por efecto de las consecuencias jurídicas negativas previstas para quienes incumplen el deber de votar. El impacto positivo del voto obligatorio en la participación electoral varía en función de la intensidad de la sanción legalmente establecida para castigar el incumplimien- 
to del deber de votar. Esta puede oscilar entre la mera declaración simbólica, sin consecuencias jurídicas (Costa Rica y Grecia), el pago de una multa o sanción pecuniaria (Australia y Brasil) y la restricción de derechos o capacidades jurídicas, como la prohibición de ejercer funciones públicas (Argentina), contratar con el Estado y cobrar mesadas pensionales (Uruguay) o realizar trámites ante oficinas públicas (Perú) (Flórez, 2017).

Blais (2000: 27) estimó el efecto promedio del voto obligatorio en todas las democracias en un 11 \% de participación adicional. Díaz, Gómez y Useche (2017: 51) encontraron una tasa de participación electoral un 23,4 \% superior en las democracias con voto obligatorio respecto de aquellas en las que es voluntario.

Las elecciones en domingo y la edad mínima para poder votar también funcionan como predictores. Aunque, en principio, las elecciones dominicales facilitan la votación (Perea, 2002: 654), Blondel et al. $(1996,1998)$ señalan que pueden carecer de impacto si los electores privilegian sus actividades de descanso. Por su parte, cuanto más baja es la edad exigida para sufragar, menor es la participación. Esta se ve reducida en casi dos puntos cuando la edad mínima exigida para sufragar se reduce en un año (Blais, 2000: 27), debido a la apatía juvenil típica hacia las elecciones.

\section{POR QUÉ EL VOTO OBLIGATORIO NO ES LA SOLUCIÓN AL «PROBLEMA» DEL ABSTENCIONISMO}

La cacería de brujas en que se ha convertido la «lucha contra el abstencionismo» en muchas democracias, que estigmatizan como «malos ciudadanos» a las personas que no votan, es la consecuencia de la incomprensión del fenómeno del voto.

Esa satanización parte de la falsa premisa de que las personas están obligadas a interesarse por la política electoral ${ }^{4}$ y de que la mayor participación electoral es necesariamente un signo de robustez democrática. Esa creencia espuria ha conducido a que varios países le apuesten a la creación de "«ciudadanos a la fuerza», mediante incentivos de voto obligatorio ( $\mathrm{VO}$, en lo sucesivo) más o menos draconianos.

Desde hace años se discute en varias democracias del mundo la posibilidad de experimentar con el VO. Se trata de un debate importante, pero que conviene

4 Un buen ejemplo de ese enfoque se encuentra en el estupendo libro de Vallejo (2016). La puesta en entredicho del argumento del «buen ciudadano interesado en política» se puede leer en el prólogo de Flórez (2016) al mismo libro. 
dar sin caer en la candidez de pensar que el sufragio obligatorio sería la panacea para la democracia, ni tampoco en el catastrofismo de afirmar que conduciría a su ruina.

La obligatoriedad del sufragio puede ofrecer algunas ventajas, pero también plantea dificultades que hay que considerar para poder diseñar un incentivo adecuado. La principal virtud del VO es que tendría un impacto inmediato en la reducción del abstencionismo, aumentando con ello la legitimidad democrática tanto del sistema político como de sus gobernantes.

Lo anterior, desde luego, si se asume como medida de la legitimidad de un gobierno solamente la cantidad de votos emitidos para elegirlo, con independencia de la calidad de esos votos. En caso de que se consideren otras características del voto, como por ejemplo lo informado y en consecuencia lo representativo que resulta de las preferencias electorales, se puede llegar a la conclusión opuesta: que el VO empobrece la calidad de la democracia y reduce la legitimidad de los gobiernos.

Fuera de la evidente pérdida de libertad que la implementación del VO supone para los ciudadanos, el aumento de la participación que induce podría tener un costo muy alto en términos de su calidad. De ahí que Rosema (2007) plantee, con suspicacia, que el abstencionismo generalizado podría ser una «bendición disfrazada» para la democracia, en la medida en que la voluntariedad del voto hace que participen personas con mayores ingresos y, en virtud de ello, mejor educadas, así como electores mayores y, por lo tanto, políticamente más maduros.

Más recientemente, Singh (2016) estableció empíricamente en un análisis transnacional que, al movilizar a la fuerza a votantes inexpertos, políticamente desinteresados y que ven los comicios como algo defectuoso o inútil, uno de los «efectos secundarios» del VO es volver las elecciones menos susceptibles de reflejar las preferencias de la población votante y hacerlas producir decisiones electorales menos significativas. Eso se debe en gran medida a que los electores que votan forzados se informan menos para hacerlo, independientemente de su nivel educativo (Singh y Roy, 2018).

El principal dilema que plantea la implementación del VO es, entonces, entre una mayor participación electoral y su menor calidad, en términos de lo informada que resulta la decisión electoral. Por esa razón, antes de formar nuestra opinión al respecto, deberíamos hacernos al menos tres preguntas:

1. ¿Cómo evitar que el VO, al movilizar especialmente a los segmentos sociales de menor ingreso, dispare la compraventa de votos? A ese respecto Becerra (2015) pronostica, desde una perspectiva meramente teórica (sin 
evidencia empírica), el efecto opuesto: «La obligación de votar elevaría de tal manera el precio del voto en el mercado negro que, incluso, podría hacer inviable su compra desde el punto de vista económico, lo cual redundaría en una mejora sustancial de la democracia».

2. ¿Es preferible que alrededor de la mitad del electorado no vote, con tal de que quienes lo hagan estén movidos por un verdadero interés de expresar sus preferencias políticas, en lugar de por el mero miedo a sufrir una sanción, que hace que el elector improvise una decisión electoral menos informada?

3. O, por el contrario, como el abstencionismo afecta fundamentalmente a personas políticamente excluidas (pobres, poco educadas y jóvenes), ¿el VO podría ser una medida eficaz para incluirlas en el sistema político, aunque sea a la fuerza, y empezar a formar ciudadanos más activos y funcionales?

Esas preguntas trazan la hoja de ruta para futuras investigaciones sobre los efectos esperados e inesperados del sufragio obligatorio. Dependiendo de las respuestas que les demos a esos interrogantes, podremos inclinarnos a apoyar o desaconsejar el VO. Bienvenido sea el debate, pero racional e informado.

La "fiebre del voto obligatorio" podría estar conduciendo a empobrecer la calidad de la participación electoral en las democracias que lo instrumentan. Si el votante promedio se encuentra profundamente desinformado, «¿deberíamos forzar a los borrachos a conducir?» (Brennan y Hill, 2014: 83).

\section{CONCLUSIÓN GENERAL}

Según la evidencia disponible, el voto es un fenómeno multicausal que responde a variables tanto individuales como contextuales que reducen o aumentan la proclividad a votar de los ciudadanos. La magnitud del abstencionismo electoral depende de múltiples factores, dentro de los cuales el voto obligatorio se destaca como el factor institucional más fácilmente manipulable y más efectivo para aumentar la participación electoral, en función de la intensidad de las sanciones previstas en los ordenamientos jurídicos para castigar el incumplimiento del deber de votar.

El debate sobre la conveniencia del voto obligatorio tiene que ver entonces con la definición misma de democracia porque especifica el tipo de ciudadano que se busca construir en los espacios democráticos. Si se prefiere construir «ciudadanos a la fuerza» con tal de reducir el abstencionismo, obligar a las personas a votar cobra sentido, a pesar de que sus preferencias electorales 
serán menos significativas como consecuencia de que los votantes movilizados por la amenaza de una sanción tienden a estar menos informados e interesados en la política que aquellos que votan espontáneamente.

Por el contrario, si se quiere privilegiar una perspectiva democrática liberal, en la que el voto es el resultado de un proceso educativo y voluntario de construcción de ciudadanía y apoyo consensuado al sistema democrático, carece de sentido que las personas sean forzadas a votar porque su voto se convertiría en una limitación de su libertad en lugar de un verdadero canal de expresión de sus preferencias políticas, dentro de las cuales, la primera de ellas debería ser la decisión voluntaria de participar en política electoral mediante el voto o la posibilidad de abstenerse de hacerlo. Desde esa perspectiva, se puede considerar que el abstencionismo es una especie de «filtro natural» que hace que solo los ciudadanos mejor informados voten y opera como una suerte de «bendición disfrazada» (Rosema, 2007) para la democracia porque mejora la calidad de la participación electoral.

\section{REFERENCIAS}

Abitbol, Pablo y Felipe Botero (2005), “Teoría de elección racional: estructura conceptual y evolución reciente", Colombia Internacional, núm. 62, julio-diciembre, pp. 132145.

Ansolabehere, S. et al. (1999), "Replicating experiments using aggregate and survey data: the case of negative advertising and turnout", American Political Science Review, vol. 93, núm. 4, pp. 901-909.

Ansolabehere, S. et al. (1994). "Does attack advertising demobilise the electorate?", American Political Science Review, vol. 88, núm. 4, pp. 829-838.

Becerra, Germán (2015). El precio del voto y la democracia, certificado de Registro de Obra Literaria Inédita núm. 10-531-177 del 26 de agosto de 2015.

Blais, André (2011), "Political Participation", en Lawrence Le Duc, Richard Niemi y Pippa Norris (eds.), Comparing Democracies 3: Elections and Voting in the 21st Century, Londres, SAGE, pp. 165-184.

Blais, André (2006), “What Affects Voter Turnout?”, Annual Review of Political Science, núm. 9, pp. 111-125.

Blais, André (2000), To Vote or Not to Vote? The Merits and Limits of Rational Choice Theory, Pittsburgh, University of Pittsburgh Press. 
Blais, André y R. Kenneth Carty (1990), “Does proportional representation foster voter turnout?", European Journal of Political Research, vol. 18, núm. 2, pp. 167-181.

Blais, André y A. Dobrzynska (1998), “Turnout in electoral democracies”, European Journal of Political Research, vol. 33, núm. 2, pp. 239-261.

Blondel, Jean et al. (1998), People and Parliament in the European Union, Oxford, Clarendon Press.

Blondel, Jean et al. (1996). "Institutions and attitudes: Towards an understanding of the problem of low turnout in the European Parliament elections of 1994", European University Institute Working Papers 96/19, Florence: European University Institute.

Brennan, Jason y Lisa Hill (2014), Compulsory Voting. For and Against, Nueva York, Cambridge University Press.

Chapman, R. G. y K. S. Palda (1983), “Electoral turnout in rational voting and consumption perspectives", Journal of Consumer Research, vol. 9, núm. 4, pp. 337-346.

Cohen, G. (1982), "Community cohesion and space planning”, en R. Frankenberg (ed.), Custom and Conflict in British Society, Manchester, Manchester University Press.

Coppock, A. y D. Green (2015), "Is voting habit forming? New evidence suggests that habitformation varies by election type", Paper presented in American Political Science Association, Chicago, Illinois.

Cox, G. W. y M. C. Munger (1989), “Closeness, expenditures and turnout in the 1982 US house elections", American Political Science Review, vol. 83, núm. 1, pp. 217-230.

Dawson, P. A. y J. E. Zinser (1976), "Political finance and participation in congressional elections", Annals of the American Academy of Political and Sociological Science, núm. 425, pp. 59-73.

Díaz, Tatiana et al. (2017), El voto obligatorio en el mundo, Tesis de Maestría en Derecho con énfasis en Derecho Público, Bogotá, Universidad Externado de Colombia, dirigida por José Fernando Flórez.

Downs, Anthony (1957), An Economic Theory of Democracy, Nueva York, Harper and Row.

Flórez, José Fernando et al. (2018), El pueblo contra sí mismo: el plebiscito por la paz en Colombia y los límites de la democracia, Bogotá, Ediciones Nueva Jurídica/Fondo de Publicaciones Corporación Universitaria Republicana. 
Flórez, José Fernando (2018a), Voter sans élire: le caractère antidémocratique de la réélection présidentielle immédiate en Amérique Latine, 1994-2018, París, Institut Universitaire Varenne Collection des Thèses.

Flórez, José Fernando (2018b), “El efecto antidemocrático de la reelección presidencial inmediata en América Latina, 1994-2017", en Magdalena Correa y Paula Robledo (eds.), Diseño institucional del Estado democrático en América Latina, Bogotá, Universidad Externado de Colombia, pp. 19-88.

Flórez, José Fernando (2017), “El dilema del voto obligatorio”, El Tiempo, 7 de marzo.

Flórez, José Fernando (2016), "La libertad como único fundamento de la libertad", Serie Documentos de Trabajo núm. 71, Bogotá, Universidad Externado de Colombia, Departamento de Derecho Constitucional.

Geys, Benny (2006), "Explaining voter turnout: A review of aggregate-level”, Electoral Studies, vol. 25, núm. 4, pp. 637-663.

Goerres (2007), "Why are Older People More Likely to Vote? The Impact of Ageing on Electoral Turnout in Europe, The British Journal of Politics and International Relations, vol. 9, núm. 1, pp. 90-121.

Hansen, T. (1994), "Local elections and local government performance”, Scandinavian Political Studies, vol. 17, núm. 1, pp. 1-30.

Healy, Andrew et al. (2010), "Irrelevant Events Affect Voters' Evaluations of Government Performance", PNAS, vol. 107, núm. 29, p. 12804-12809.

Healy, Andrew et al. (2009), “Euphoria and Retrospective Voting: The Impact of College Football Outcomes on Incumbent Reelection", Paper presented at the annual meeting of the Midwest Political Science Association 67th Annual National Conference, The Palmer House Hilton, Chicago, IL, 2 de abril.

Hoffman-Martinot, V. (1994), “Voter turnout in French municipal elections”, en L. Lopez-Nieto (ed.), Local Elections in Europe, Barcelona, Institut de ciènces politiques I socials, pp. 13-42.

Holbein, John (2017), "Childhood Skill Development and Adult Political Participation", American Political Science Review, vol. 111, núm. 3, pp. 572-583.

International Institute for Democracy and Electoral Assistance (IDEA) (2013), Voter Turnout Database.

Jackman, R. W. (1987), "Political institutions and voter turnout in the industrial democracies", American Political Science Review, vol. 81, núm. 2, pp. 405-423. 
Jacobs, Jane (1969), The Economy of Cities, Nueva York, Random House.

Kostadinova, Tatiana y Timothy Power (2007), "Does Democratization Depress Participation? Voter Turnout in the Latin American and Eastern European Transitional Democracies", Political Research Quarterly, vol. 60, núm. 3, pp.363-377.

Ladner, A. y H. Milner (1999), "Do voters turn out more under proportional than majoritarian systems? The evidence from Swiss communal elections", Electoral Studies, vol. 18, núm. 2, pp. 235-250.

La Silla Vacía (2014), “Un triunfo de la selección = 300 mil votos más para Santos”, 11 de junio.

Lehingue, Patrick (2011), Le vote. Approches sociologiques de l'institution et des comportements électoraux, París, La Découverte.

Lehoucq, F. y D. L. Wall (2004), “Explaining Voter Turnout Rates in New Democracies: Guatemala", Electoral Studies, vol. 23, núm. 3, pp. 485-500.

Leighley, Jan E. y Jonathan Nagler (2013), Who Votes Now? Demographics, Issues, Inequality, and Turnout in the United States, Princeton, Princeton University Press.

Lijphart, Arend (1997), “Unequal Participation: Democracy's Unresolved Dilemma”, American Political Science Review, vol. 91, núm. 1: pp. 1-14.

Maldonado, Arturo (2011), "Compulsory voting and the decision to vote", Americas Barometer Insights, núm. 63, pp.1-9.

Navia, Patricio y Rodrigo Osorio (2015), “Una aproximación al estado del voto económico en América Latina Política", Revista de Ciencia Política, vol. 53, núm. 1: pp. 7-14.

Percival, Garrick et al. (2007), "Taxing, Spending, and Voting: Voter Turnout Rates in Statewide Elections in Comparative Perspective", State and Local Government Review, vol. 39, núm. 3, pp. 131-43.

Perea, Eva (2002), “Individual characteristics, institutional incentives and electoral abstention in Western Europe", European Journal of Political Research, vol. 41, núm. 5, pp. 643-673.

Persson, Anna y Bo Rothstein (2015), "It's My Money: Why Big Government May Be Good Government", Comparative Politics, vol. 47, núm. 2, enero, pp. 231-249.

Power, T. J. y J. T. Roberts (1995), “Compulsory voting, invalid ballots, and abstention in Brazil", Political Research Quarterly, vol. 48, núm. 4, pp. 795-826. 
Rojas, Hernando y Jill Hopke (2010), "Socializados para la autocensura: comunicacio?n autoritaria y opinio?n pu?blica", Revista Latinoamericana de Opinión Pública, vol. 1, núm. 0 .

Rosema, Martin (2007), "Low turnout: Threat to democracy or blessing in disguise? Consequences of citizens' varying tendencies to vote", Electoral Studies, vol. 26, núm. 3, pp. 612-623.

Seidle, L. y D. Miller, (1976), "Turnout, rational abstention and campaign effort", Public Choice, vol. 27, núm. 1, pp. 121-126.

Singh, Shane y Jason Roy (2018), "Compulsory voting and voter information seeking", Research and Politics, vol. 5, núm. 1, pp.1-8.

Singh, Shane (2016), "Elections as poorer reflections of preferences under compulsory voting", Electoral Studies, vol. 44, núm. 1, pp. 56-65.

The New York Times (2014), "World Cup Opinions in 19 Countries: Likes, Dislikes, Predictions", 10 de junio.

Topf, Richard (1995), "Electoral participation", en Hans-Dieter Klingemann \& Dieter Fuchs (eds.), Citizens and the State, Oxford, Oxford University Press, pp. 27-51.

Tufte, Edward (1978), Political Control of the Economy, Princeton, Princeton University Press.

Vallejo, César (2016), La política como fundamento de la libertad, Bogotá, Universidad Externado de Colombia.

Wirth, Louis (1938), "Urbanism as a way of life”, American Journal of Sociology, vol. 44, núm. 1, pp. 3-24.

Wolfinger, Raymond y Steven Rosenstone, 1980. Who Votes?, New Haven, Yale University Press. 